\title{
Industry Perceptions of Graduate Attribute COMPETENCIES
}

\author{
Donald S. Petkau \\ Department of Biosystems Engineering, University of Manitoba, Winnipeg, MB, R3T 5V6 \\ Don.Petkau@umanitoba.ca
}

\begin{abstract}
Through the CEAB accreditation program, graduate attribute competencies are currently being standardized throughout the engineering programs in Canada. Challenges of these requirements are in understanding the implementation process and determining the outcome levels of the students. This study went outside of the university environment to determine the outcome levels found in graduated students. It consisted of a series of interviews with two groups of engineers working in a major energy corporation in the Province of Manitoba. One group consisted of senior engineers with a minimum of 15 years experience while the other was of new graduates with at minimum 18 months of service. The groups were asked questions on job requirements for new graduates with a follow-up survey on levels of competency for each graduate attribute. This paper describes the process and the analysis of the information. Results were consistent between both groups but show levels of competency at lower levels than determined from a student perspective at time of graduation.
\end{abstract}

Keywords: Graduate attributes, Engineering competency, Student perspective

\section{INTRODUCTION}

Engineering schools in Canada are required to assess graduates of their programs according to specified graduate attributes in order to gain accreditation. At the University of Manitoba the assessment involves direct methods (assessing coursework to the Canadian Engineering Accreditation Board (CEAB) graduate attributes) and indirect methods including surveys and forums. Typically most of the data is collected from students at time of graduation with a self assessment process. The specific purpose of this paper is to describe my $\mathrm{PhD}$ research which included an in-depth study of the engineering competencies required in an engineering workplace.

The research partner in this project was Manitoba Hydro, a Provincial Crown Corporation that provides hydro electric energy and natural gas to customers throughout Manitoba. They have expressed concern that as some engineering designers complete their careers, the organization loses valuable knowledge. They are struggling to bring their junior engineers to a point of meaningful contribution to their engineering process. Their goal is to hire design ready engineers or to develop methods to educate junior engineers more quickly. A cooperative plan was developed to study engineering designers at Manitoba Hydro.

There is little information on how a newly graduated engineer's education relates to the workplace and what additional learning takes place subsequent to their formal education. A determination of these factors could lead to the better understanding of whether this knowledge could be incorporated into an engineering educational program. This may also determine that this knowledge is more suited to a training program undertaken in the workplace during the first years of an engineers' career.

Currently we use a series of graduate attributes as determined by the accreditation program in Canada (Engineers Canada 2011) [2] to help determine the qualifications of a graduating engineer. These attributes are a definition of qualifications for engineering graduates upon their completion of an accredited program. The question is how these attributes define the capabilities of a new graduate. There is an understanding that a new graduate is not considered a professional until they have completed a further four years of mentorship under the tutelage of an experienced professional engineer. They must first complete a number of stages of development as required by the Association of Professional Engineers and Geoscientists of the Province of Manitoba (APEGM). Engineering graduates must complete a four year term as an Engineer in Training (EIT) prior to receiving their professional engineering designation (P.Eng). This program includes performance of engineering work under the supervision of a professional engineer, individual development opportunities, and exams in engineering practice and ethics. This is an indication that engineering graduates must gain additional skills and knowledge in the workplace after graduation. These are not currently defined and it may be difficult to determine this additional 
knowledge as each engineering position within each organization will require a different knowledge basis.

What are the additional qualifications an engineering graduate must attain subsequent to their formal engineering education? How do these qualifications provide a link between the formal education of an engineer and the practice of a professional engineer?

The primary objective of this research was to define how the graduate attributes used in our engineering education programs link to the practice of professional engineering.

The specific objectives to achieve this goal were:

1. identify the role of an expert or professional engineer

2. identify the role of a novice or newly graduated engineer

3. identify the knowledge of a newly graduated engineer with respect to the graduate attributes

4. identify the knowledge requirements of the workplace for a newly graduated engineer with respect to the graduate attributes

5. determine the link and/or gaps between these abilities and knowledge requirements of newly graduated engineers to that of a professional engineer.

This paper will summarize the results of the perception of senior engineers and new graduates engineers on the competency level of new graduates with respect to the $\mathrm{CEAB}$ graduate attributes.

\section{RESEARCH METHODOLOGY}

\subsection{Background}

This study is a quantitative descriptive study characterizing engineering experiences within the work environment using descriptive statistics with a series of mixed method data collection tools. This study was of an exploratory nature using a sequential data collection method. The first stage was in the collection of engineering task related information using an open ended interview style. A total of three interviews were conducted with two sets of participants. The first set was a group of professional engineers in senior management positions. The second set of participants was a group of newly graduated engineers in their first three years of employment. The interviews were designed to gather information on the job tasks of each group of engineers. The senior engineers were also interviewed on the required job tasks of the newly graduated engineer.

The second data collection technique was the use of questionnaires. A total of three questionnaires were completed by each participant. The first one was on the aspects of engineering practice which were correlated to the participants' job tasks compiled from the interviews. The second questionnaire was on the graduate attributes and correlated engineering education to the job tasks of a newly graduated engineer. The third questionnaire was also on the graduate attributes but this one evaluated the strengths and weaknesses of the new graduate.

This study took place in the offices of Manitoba Hydro which is a Crown Corporation in the province of Manitoba. It is the province's major energy utility supplying electricity and natural gas to customers throughout Manitoba. It also supplies electricity to other markets in Canada and the mid-western United States. The corporation has more than 6000 employees and operates out of numerous locations in Winnipeg and throughout the province of Manitoba.

When selecting the appropriate participants it was important to maintain variability in the sample as well as to eliminate bias in the selection methods. The size of this corporation and number of engineering departments allowed for potential variability. The type of study would restrict the number of participants involved. In order to be able to generalize results participants were chosen from a number of engineering disciplines. Manitoba Hydro has a variety of engineering disciplines on staff. Another important factor for recruitment was the availability and cooperation of the participants. The main recruitment criteria were the years of professional experience.

\subsection{Participants}

There were two separate groups of participants in this research study. The first was a group of four recent graduates from the engineering program at the University of Manitoba. They were all enrolled in the EIT program at APEGM and are referred to as EIT's in this study. This was a diversified group with two electrical engineers, one mechanical engineer, and one civil engineer. They had graduated from their programs from between fourteen months earlier to the longest at three years earlier. One of the individuals had no previous engineering experience even in a summer job. The other three individuals had been working in engineering related summer jobs prior to graduation. Two had worked for Manitoba Hydro in the past and so had additional experience in regards to some of the orientation and cultural aspects of the corporation. Three of the four individuals were currently in or had gone through the EIT program at Manitoba Hydro. This program allows new graduates to spend six months working in a particular area of the corporation. At the completion of their six month term they choose another department for the following six months. The EIT's are allowed to complete up to four of these terms prior to their permanent placement. The one individual who had not entered through this EIT program had spent a summer term in a particular department and upon graduation continued in the same role as a permanent employee. This information is relevant to the study as the individuals in the EIT program spend the first two years of 
employment in a situation equivalent to four new positions. This affects their job role as they need additional training and orientation with each of these placements. This could affect this study as individuals are involved with more entry level activities rather than a steady progression to increased responsibilities. This should not have a major affect though as only one of the individuals is currently in the EIT program. All of the other three participants are in permanent placements with two of them already in the second year of their positions.

The second set of participants was a group of professional engineers and are referred to as P.Eng's in this study. All of these individuals have spent their careers with Manitoba Hydro and have had their professional engineering status from between nine to twenty-two years. These engineers are all in supervisory positions. They are currently supervising EIT's or have done so on numerous occasions in the past. All four of the professional engineering participants were electrical engineers.

\subsection{Ethical And Confidentiality Considerations}

This is a study involving individuals employed by a large corporation. Anytime people are part of a study considerations are required to protect the individuals. This is especially important for this study as interview information is requested from the participants on their engineering tasks and how these tasks are performed. Another consideration is the fact that all participants are employed by the same corporation. Some of the participants in the pilot study had supervisory/employee relationships. It was important to protect information exchanged and to eliminate the prospect of any coercion to participate. These issues were dealt with through appropriate ethical approval procedures. Anonymity measures were undertaken with all data sources including individual interview, focus groups, and questionnaires. Participants were never identified and data was coded to strip any identification prior to any publication. Consideration was taken that there was no position of power between members of the senior engineering group and the junior engineering group. All ethical issues were covered by signed agreements between participants and the researcher. The protocol for the project was approved by the University's research ethics board.

An issue of confidentiality for this project was of a corporate nature. Manitoba Hydro was concerned that information detrimental to the corporation expressed during an interview could be released by the researcher. An agreement was reached with a signed contract between the corporation and the researcher to protect both parties.

\subsection{Survey Instrument}

The survey document was modified from the survey used by Cicek [1] in a survey of fourth year students at the University of Manitoba. The intent was to use the same instrument used on graduating students to survey new engineers after a number of years on the job. The modifications to the instrument, shown in Figure 1, were in the wording of the document. Since the participants were now in the work force they were asked to evaluate their educational knowledge at the time of entry to the workforce. They were also asked to evaluate the level of educational knowledge required to complete their work tasks at the time of entry to the workforce. The senior participants were also asked to evaluate the new employees in the same manner. They were instructed to give a general evaluation of the new graduates that they had supervised.

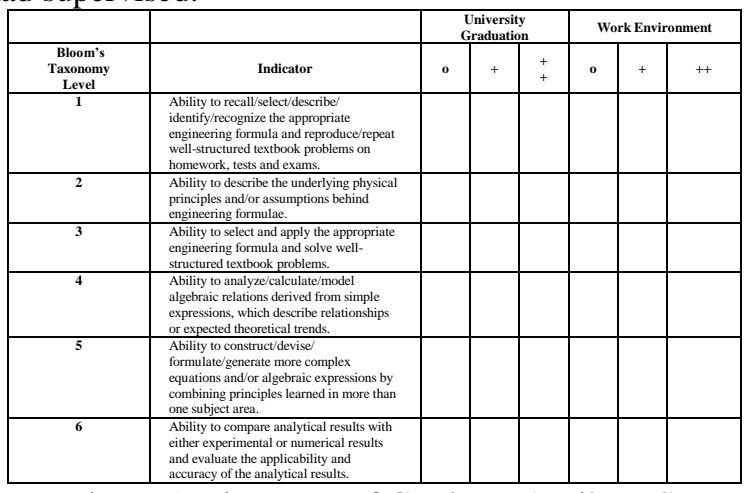

Figure 1: First page of Graduate Attribute Survey

An additional survey instrument, shown in Figure 2, was also modified from one used by Cicek [1]. This asked the new graduates to rank the graduate attributes according to which was the strongest in their engineering education and which was the most important to the workplace. The senior participants were also asked to make this ranking based on their experience with new graduates and on which attribute was the most important for a new graduate upon entry to the workplace.

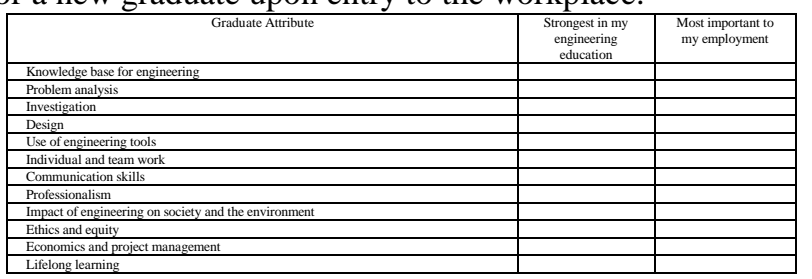

Figure 2: Last page of Graduate Attribute Survey for ranking attributes

\section{RESULTS AND DISCUSSION}

\subsection{Results of EIT's Survey}

There were four EIT's which participated in both the survey and the interview portions of the study. A 
numerical value of " 1 " was assigned if the EIT's indicated that the skill/ability had not been developed, a value of " 2 " was assigned if the skill/ability had been introduced but not mastered and a value of " 3 " was assigned if the skill/ability was indicated as mastered. The results of their perceived competencies at time of entry to the workplace are summarized in Figure 3.

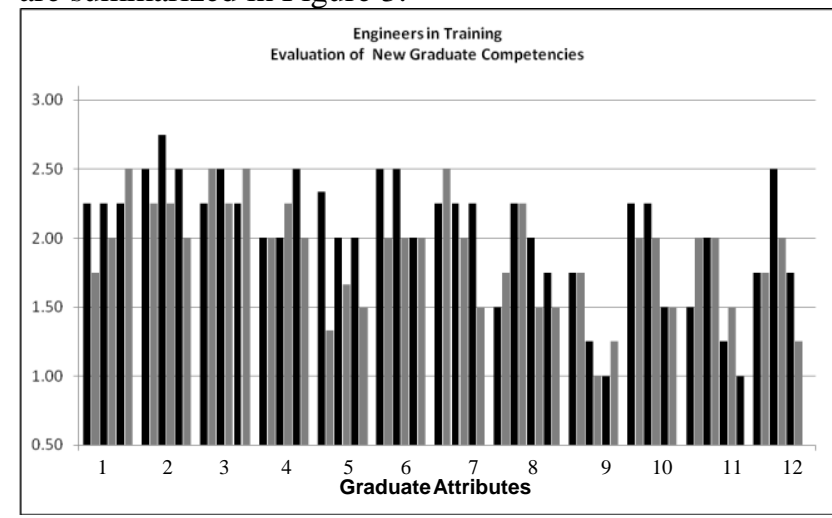

Figure 3: Average perceived graduate competencies of new engineering graduates by EIT.

The overall results show some similarities and differences when compared to results from studies of new graduates' perceived competencies in the Cicek [1] study. A major difference is that new graduates assessed an overall average value of 2.55 in the Cicek study, compared to a value of 1.98 after engineering graduates had been in the workforce for a period of time. From this study results show that the participant's assessment of graduating students was that they have an average skill/ability level that would be considered only at the introduced level. Overall they also felt that half the graduate attributes were even barely introduced. This information was backed up by the interview portion of the study. Data from the interviews was also compiled and analyzed to correlate with the survey data but this information did not yield results as discussions only cover certain attributes. Individuals would discuss work tasks they felt important and therefore half of the twelve attributes would have no data. The valuable information from the interviews came from the confirmation of the challenges and importance of the attributes during the first few years in the workforce. Discussions in the interviews were on how they struggled with understanding their role in the workplace and on how they wished they had received additional training in areas they felt they were unprepared for the workplace.

The EIT participants rated the attributes of 2-Problem Analysis, and 3-Investigation as the highest for their skill/ability levels with an average rating of 2.38. New graduates in the Cicek [1] study rated 6-Individual and Team Work as the highest rated attribute along with 1Knowledge Base for Engineering, 7-Communication, and 12-Lifelong Learning with each of these attributes receiving a rating of above 2.50 . In this study the EIT participants only rated six of the graduate attributes above a rating of 2.00 and four of these ranged from 2.13 to 2.17. This indicates that these participants only considered two of the attributes to have rated as above an introduced level. The six remaining attributes rated from a level of 1.33 to 1.92 . The lowest two attributes were 9-

Impact on Society and Environment and 11-Economics and Project Management. Information from the individual interviews backed up this information as it was indicated that they felt unprepared for the project management role they were asked to undertake in their work.

Looking at each of the graduate attributes individually there are some interesting observations. Having used the survey based on Bloom's Taxonomy, expectations were that skill/ability level would be highest in the lower levels and decrease with movement from the knowledge level to the evaluation level. This was not the case as in some instances the knowledge and comprehension levels were rated low while the synthesis and evaluation levels were rated higher. In the more technical attributes such as 1Knowledge Base for Engineering, 2-Problem Analysis, and 3-Investigation, the ratings were fairly evenly distributed over all of the Blooms levels. This could indicate that the EIT's were comfortable with their skill/ability level. This was confirmed by the interview portion of the study as individuals indicated they spent considerable time working in these areas and had sufficient knowledge to manage the work tasks. Other attributes such as 5-Engineering Tools, 8Professionalism, 9-Impact on Society and the Environment, 10-Ethics, 11-Project Management, and 12-Lifelong Learning had a much wider range of ratings within the Bloom's levels with some inconsistency. There are some explanations from the interviews with discussion on a lack of understanding in the areas of professionalism, and ethics. The participants had a more difficult time describing or defining these attributes. This made it more difficult for them to give an evaluation with any confidence.

\subsection{Results of P.Eng Survey}

There were four P.Eng's which participated in both the survey and the interview portions of the study. A numerical value of " 1 " was assigned if the P.Eng indicated that the skill/ability had not been developed by a new graduate upon entry to the workplace, a value of "2" was assigned if the skill/ability had been introduced but not mastered and a value of " 3 " was assigned if the skill/ability was indicated as mastered. The results from the P.Eng ratings (1.96), as seen in Figure 4, had the same 
overall average as that of the EIT's (1.98) but the distribution of results was much different.

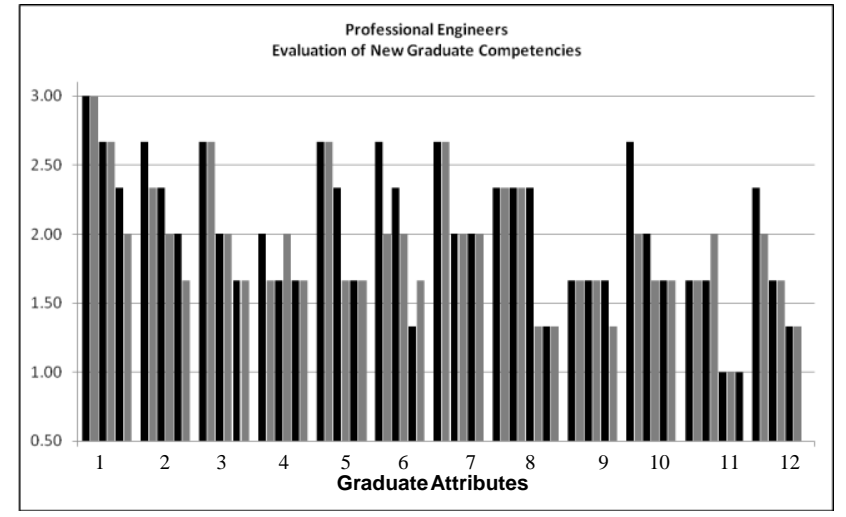

Figure 4: Average perceived graduate competencies of new engineering graduates by P.Eng.

The senior group in rating new graduates coming into the workplace had a much more consistent ranking with respect to Blooms level. They consistently ranked the lower levels of knowledge and comprehension as high with the upper levels of synthesis and evaluation as much lower. The attributes 1-Knowledge Base for Engineering, 2-Problem Analysis, and 7Communication were the top rated attributes with average ratings between 2.17 and 2.61. This compares to the EIT ratings of 2.38 for 2-Problem Analysis and 3Investigation. While they are not exactly the same they are in the more technical end of the attributes. The lowest rated attributes by the P.Eng's were 9-Impact on Society and the Environment and 11-Economics and Project Management with ratings between 1.39 and 1.61. This compares to the EIT ratings ranging from 1.33 to 1.63 on the same two attributes. There was agreement from both sets of participants on where the new graduate weaknesses were on entry to the workplace. Interview discussions with the P.Eng participants showed that they felt there were some attributes that new graduates would not necessarily need detailed knowledge coming into the workplace. In the attributes 9-Impact on Society and the Environment and 11-Economics and Project Management they said that new graduates would not be expected to had much prior knowledge and would be expected to learn these aspects on the job.

In examining a comparison of the overall results by both groups in Figure 5 trends are very similar. There are only slight differences in the first four attributes. The P.Eng participant's felt that the new graduates have higher capabilities in 1-Knowledge Base for Engineering and a slightly lower assessment in 2-Problem Analysis, 3Investigation, and 4-Design. Some of these differences were discussed in the interviews. The P.Eng group was pleased with the work of the new graduates in these areas and so would assess them favourably. The EIT group stated that at times they were overwhelmed with their tasks and so could feel that their skills/abilities were less than required.

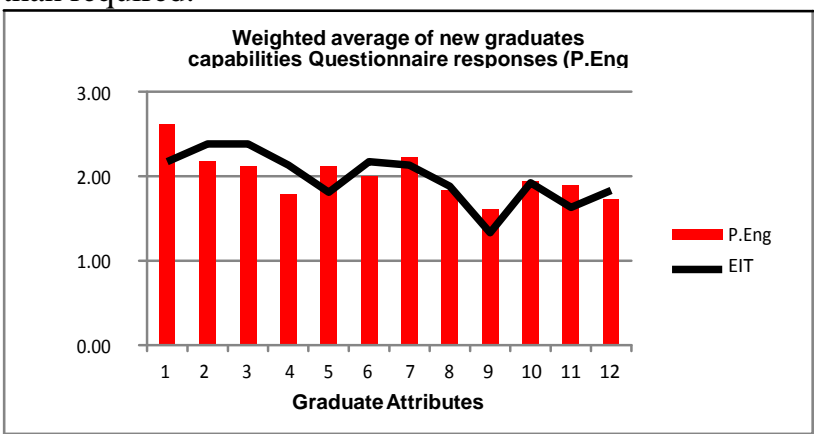

Figure 5: Comparison of average perceived graduate attribute competencies for new engineering graduates (P.Eng vs EIT).

\subsection{Results of Ranking the Graduate Attributes}

The final data collected from the participants on the graduate attribute questionnaire was a ranking of the attributes. This was done in two ways, the first being as to the strongest in skill level for the new graduate and second in the most important to the workplace. The P.Eng rankings followed the list of the graduate attributes almost exactly. The top ranked one was 1-Knowledge Base for Engineering, second ranked was 2-Problem Analysis, third ranked was 3-Investigation and so on. The only attribute that did not follow this pattern was 4Design, which was bumped up to seventh place. It was observed that the P.Eng group felt that the strengths of the new graduate were in the technical graduate attributes. The attributes for the soft skills were considered to be the weaker skills for new graduate competencies.

Looking at the EIT results they do not assign rankings quite as consistently with the list of graduate attributes. Even so they are not that far removed from the rankings by the professional engineers. The observation for the EIT group is that the spread in the rating values is much smaller than that of the P.Eng group. These are averages of each individual's ratings so this low spread would indicate more variability between the individual ratings. The major difference in the two groups rankings is that the EIT's classify the attribute 6-Individual and Team Work as one of the most important the attribute 9-Impact on Society and the Environment as substantially lower than all the other attributes.

\subsection{Limitations of This Mixed Method Study}

This study was a mixed method study using interviews and questionnaires. The use of the interviews in this, a quantitative study, allowed for a real depth of understanding of not only what but how engineers 
complete activities in the workplace. This method allowed the researcher and the participant to reflect on what they do each day and provided ability for them to give detailed descriptions. Another advantage of using these two methods is the ability to triangulate the data. Information from the interviews was used to enhance the activity descriptions and to cross reference with the results of the questionnaire.

There are also disadvantages to using interviews in this study. The use of interviews limits the size of the participant group in two ways. First, because of the time involved and amount of data collected, there is a limit to the size of the participant group. Secondly, for the same reasons, it limits who is available and who is willing to participate. The limitation of the size of the participant group restricts the results to this particular group. Some of the results could be indicative of the Manitoba Hydro organization. Due to culture and structure, certain activities would be common. Other aspects would be more representative of the individual's personality and of their department. Results would be difficult to take outside of the organization due to differences in organizational culture, structure, type of work, and type of organization.

\section{CONCLUSIONS}

This was an exploratory study of engineers in the workplace to form a basis on how engineering education translates to the activities in the workplace. It was conducted as a mixed method study within a large energy corporation in the Province of Manitoba. A number of professional engineers and engineers in training were interviewed and asked to complete questionnaires. The questions were developed to determine the role of engineering education in the workplace. These questions inquired into an assessment of the level of knowledge a new engineering graduate has at time of entry to the workplace.

The objective was to determine the knowledge of a newly graduated engineer and to identify the knowledge requirements of the workplace with respect to the CEAB graduate attributes. The information was compiled through the interviews and questionnaires from both P.Eng's and EIT's on the activities of newly graduated engineers.

The interview data had some limitations as discussions centered on certain graduate attributes with minimal data in the other attributes. Data was coded into the six levels of Blooms Taxonomy for each of the graduate attributes. Analysis of the interview data showed that the both the EIT and the P.Eng participants discussed the role of a new graduate as being in the comprehension and application levels of the graduate attributes. Assessment was consistent between the two groups in both the level of Bloom's Taxonomy and category of graduate attribute.
The second method of collecting information was with a questionnaire. The P.Eng's were asked to evaluate the capabilities and the job requirements for a newly graduated engineer. The EIT's were asked to give a self evaluation of their own capabilities at graduation and an assessment of their first job activities. The evaluation of the data showed consistency among the members of the groups and between the groups. It showed that the skill/ability level of new graduates was assessed at a much lower level than of graduating students self assessments as discussed by Cicek [1]. EIT's self assessment was slightly above an introduced level of knowledge. The P.Eng assessment of the EIT abilities was equal to or higher than the EIT self assessment in all graduate attributes except one. In the graduate attribute 4-Design the P.Eng assessment was slightly lower. The main difference between the two group ratings was the P.Eng assessed the knowledge levels of the EITs higher in the lower levels of Bloom's Taxonomy with progressively lower assessments in the upper levels. The EIT's self evaluation saw a random distribution of assessment through the six levels of Bloom's Taxonomy.

This study was on four professional engineers and four engineers in training within one corporation. This small sample size means that the conclusions are of a general nature and will be used as a guide for future research.

\section{References}

[1] Jill S. Cicek, Paul Labossiere and Danny Mann. "Surveying fourth year engineering student perceptions of graduate attribute competencies." In Proc of CEEA Canadian Engineering Education Conference, (Montreal, QC. June 2013)

[2] Engineers Canada, Graduate attributes, Accreditation Criteria and Procedures 2011, Canadian Engineering Accreditation Board, Accreditation Criteria and Procedures, Section 3.1 page 12. http://www.engineerscanada.ca/files/w_Accreditation_ Criteria_Procedures_2011.pdf 